Portland State University

PDXScholar

Electrical and Computer Engineering Faculty

Publications and Presentations

$10-1-2009$

\title{
Effects of Gaussian Fields on the Stability of Inhomogeneously Broadened Lasers
}

Pitak Chenkosol

Portland State University

Lee W. Casperson

Portland State University

Follow this and additional works at: https://pdxscholar.library.pdx.edu/ece_fac

Part of the Electrical and Computer Engineering Commons

Let us know how access to this document benefits you.

Citation Details

Pitak Chenkosol and Lee W. Casperson, "Effects of Gaussian fields on the stability of inhomogeneously broadened lasers," J. Opt. Soc. Am. B 26, 1837-1844 (2009).

This Article is brought to you for free and open access. It has been accepted for inclusion in Electrical and Computer Engineering Faculty Publications and Presentations by an authorized administrator of PDXScholar. Please contact us if we can make this document more accessible: pdxscholar@pdx.edu. 


\title{
Effects of Gaussian fields on the stability of inhomogeneously broadened lasers
}

\author{
Pitak Chenkosol ${ }^{1}$ and Lee W. Casperson ${ }^{2, *}$ \\ ${ }^{1}$ Department of Electrical and Computer Engineering, Portland State University, P.O. Box 751, Portland, Oregon \\ 97207-0751, USA \\ ${ }^{2}$ Department of Electrical and Computer Engineering, and Center for Optoelectronics and Optical Communications, \\ University of North Carolina at Charlotte, 9201 University City Boulevard, Charlotte, \\ North Carolina 28223-0001, USA \\ *Corresponding author: lcaspers@uncc.edu
}

Received April 15, 2009; revised July 7, 2009; accepted July 26, 2009;

posted July 29, 2009 (Doc. ID 110087); published September 4, 2009

\begin{abstract}
Under some conditions, spontaneous coherent pulsations are known to occur in the output beams of inhomogeneously broadened laser oscillators. These lasers typically operate with a Gaussian transverse field distribution, while the corresponding theoretical models assume a uniform-plane-wave field. The effects of a Gaussian field on the stability criteria of single-mode inhomogeneously broadened ring laser oscillators are considered in this study. It is found that in comparison to a plane wave a Gaussian field variation still permits low-threshold spontaneous pulsations but reduces the parameter space over which these pulsations can be observed. (C) 2009 Optical Society of America
\end{abstract}

OCIS codes: $270.3430,270.3100,020.1670,140.3460$.

\section{INTRODUCTION}

Spontaneous pulsation instabilities have been observed and studied in laser oscillators almost since the first laser was operated [1]. Most of the early theoretical studies of laser dynamics were based on rate equations that provide a mathematical description of the time-dependent planewave intensities and spatially uniform population densities, and such models were useful in interpreting many of the experimentally documented laser behaviors. The simplest rate equation laser models were, however, found to be stable, so the interpretation of laser instabilities required additional considerations or more complicated descriptions of the underlying physics. A familiar example is the spiking behavior that is observed in pulsed and $\mathrm{cw}$ ruby lasers and the difficulty in finding a satisfactory explanation.

One of the more challenging theoretical generalizations involves the abandonment of rate equations in favor of semiclassical laser models that were also developed very early. The simplest semiclassical model for a homogeneously broadened laser was shown by Haken [2] to be equivalent to the Lorenz model, which was already popular because of its prediction of unstable and chaotic behavior. However, known instabilities in homogeneously broadened lasers tend to differ in some characteristics from those predicted by the Lorenz-Haken (L-H) equations and they also tend to occur at pumping levels below the L-H instability thresholds. In an effort to make the models more realistic, the possibility of Gaussian-beam fields rather than plane waves was explored, but these field variations were found to entirely eliminate the instabilities in the simplest models [3-6]. However, it was shown that some Lorenz-like pulsation behavior in farinfrared (FIR) ammonia lasers could be modeled by gen- eralizing the L-H model to include a three-level structure and Doppler broadening $[7,8]$. In an alternate approach it was found that experimental results could be represented by generalizing the L-H model to include both a Gaussian transverse field variation and a Gaussian pump distribution $[9,10]$. More recently the models with transverse field and pump variations have been extended to homogeneously broadened lasers with multiple longitudinal modes [11-13].

The above-mentioned theoretical studies all are extensions from the L-H model for homogeneously broadened lasers. On the other hand, instabilities also exist in Lamb's Maxwell-Schrödinger model for inhomogeneously broadened gas lasers [14-20] and stability criteria for these lasers have been developed that allow for arbitrary values of the most important laser parameters [21]. For practical operating conditions, these instabilities can occur with very low thresholds and good agreement between theory and experiment has been obtained. In contrast to homogeneously broadened lasers, where extra physics must be added to obtain an approximation of experimental instability data, the theoretical interpretations for inhomogeneously broadened lasers have yielded good agreement using the simplest possible planetraveling-wave electromagnetic fields. Instead of adding physics to obtain agreement, however, one must be sure with inhomogeneously broadened lasers that no essential physics has been neglected that might undo the seemingly good agreement.

With inhomogeneously broadened lasers, some degree of spectral cross relaxation must occur due to velocitychanging collisions. It has been shown that with experimental values for the cross-relaxation rates, this effect is unimportant [22]. With homogeneously broadened lasers, 
the inclusion of standing-wave fields, as is common in practice, substantially raises the already high instability thresholds [23], but with inhomogeneously broadened lasers the thresholds remain low, and standing-wave fields were included in the first theoretical models [14]. The purpose of the present study has been to consider the possible consequences for instabilities in inhomogeneously broadened lasers when the field profile in the laser is Gaussian rather than plane wave. A comparison of type 1 perturbation stability curves [24] for both the uniformplane-wave and the constant-spot-size Gaussian-beam models shows that, at the same values of laser operating parameters, the instability tends to occur at higher pumping rates and for a narrower range of parameter values for the Gaussian-beam case than with uniform plane waves. While the increased instability thresholds and narrowed pulsation regions are of interest, the main point of these results is that in contrast to homogeneously broadened lasers there remain conditions corresponding to previous instability experiments under which spontaneous pulsations should be possible. This is reassuring, since low-threshold spontaneous pulsations are readily observed in laboratory experiments with these lasers under the predicted conditions.

The basic theoretical model for a unidirectional Gaussian beam in a uniformly pumped ring-laser oscillator is established in Section 2. The steady-state solutions of this model are also derived. A perturbation stability analysis of the model is developed in Section 3. The results of this analysis are shown graphically and compared with the corresponding results for the uniform-plane-wave model. Even with Gaussian fields, as with plane-wave fields, the pulsation thresholds for these lasers can be very close to the ordinary lasing thresholds.

\section{MODEL}

The starting point for this analysis is a set of MaxwellSchrodinger equations that has been the basis for several previous studies of laser instabilities [22]. In its reduced and normalized three-decay-rate form, this model has been the basis for a plane-wave instability study of inhomogeneously broadened gas lasers [21]. The purpose of the present study is to consider a modification of the same model that replaces the plane-wave field with a Gaussian transverse field distribution:

$$
\begin{gathered}
\frac{\partial P_{r}(V, r, t)}{\partial t}=-\gamma\left[P_{r}(V, r, t)-V P_{i}(V, r, t)\right], \\
\frac{\partial P_{i}(V, r, t)}{\partial t}=-\gamma\left\{P_{i}(V, r, t)+V P_{r}(V, r, t)\right. \\
\left.+G(r) A(t)\left[D_{0}+D(V, r, t)\right]\right\}, \\
\frac{\partial D(V, r, t)}{\partial t}=-\gamma_{d}\left[D(V, r, t)-G(r) A(t) P_{i}(V, r, t)\right], \\
\frac{\mathrm{d} A(t)}{\mathrm{d} t}=-\gamma_{c}\left[A(t)+\int_{0}^{\infty} \int_{-\infty}^{\infty} G(r) P_{i}(V, r, t) \mathrm{d} V r \mathrm{~d} r\right] .
\end{gathered}
$$

The independent variables in Eqs. (1)-(4) include the time $t$, the radius $r$, and the normalized atomic or molecu- lar velocity $V=k v / \gamma$ (for Doppler inhomogeneous broadening) where $k$ is the propagation constant, $v$ is the actual velocity, and $\gamma$ is the polarization decay rate. The dependent variables in Eqs. (1)-(4) include the real and imaginary parts of the complex polarization $P_{r}(V, r, t)$ and $P_{i}(V, r, t)$, the unsaturated population difference $D_{0}$, the saturation induced correction to the population difference $D(V, r, t)$, and the electric field amplitude $A(t)$. The function $G(r)$ was not included in the uniform-plane-wave model $[21,22,24]$ and it represents the Gaussian radial amplitude distribution. Thus the local amplitude distribution is defined by $A(r, t)=G(r) A(t)$ with $G(r)$ $=(2 / \pi)^{1 / 2} \exp \left(-r^{2} / w^{2}\right)$. The decay rates include the polarization decay rate $\gamma$, the population difference decay rate $\gamma_{d}$, and the electric field cavity decay rate $\gamma_{c}$. This formulation for inclusion of Gaussian fields is analogous to that employed previously for homogeneously broadened lasers [3-6].

Instability criteria for lasers are generally expressed in terms of the laser threshold parameter. This parameter expresses the actual pumping rate of a laser in terms of the minimum pumping required for the laser to reach the laser oscillation threshold. In the following paragraphs the threshold parameter is derived for the laser described by Eqs. (1)-(4). Considering first the laser at steady state, i.e., $\partial / \partial t=0$, Eqs. (1)-(4) reduce to

$$
\begin{gathered}
P_{r s}(V, r)=V P_{i s}(V, r), \\
P_{i s}(V, r)=-V P_{r s}(V, r)-G(r) A_{s}\left(D_{0}+D_{s}(V, r)\right), \\
D_{s}(V, r)=G(r) A_{s} P_{i s}(V, r), \\
A_{s}=-\int_{0}^{\infty} \int_{-\infty}^{\infty} G(r) P_{i s}(V, r) \mathrm{d} V r \mathrm{~d} r .
\end{gathered}
$$

Equations (5)-(7) can be combined to obtain

$$
P_{i s}(V, r)=-\frac{G(r) A_{s} D_{0}}{1+V^{2}+G^{2}(r) A_{s}^{2}} .
$$

Equations (8) and (9) then yield

$$
\begin{aligned}
A_{s}= & -\int_{0}^{\infty} \int_{-\infty}^{\infty} G(r)\left[-\frac{G(r) A_{s} D_{0}}{1+V^{2}+G^{2}(r) A_{s}^{2}}\right] \mathrm{d} V r \mathrm{~d} r \\
1 & =D_{0} \int_{0}^{\infty} G^{2}(r) \int_{-\infty}^{\infty} \frac{\mathrm{d} V}{1+V^{2}+G^{2}(r) A_{s}^{2}} r \mathrm{~d} r \\
& =\pi D_{0} \int_{0}^{\infty} \frac{G^{2}(r) r \mathrm{~d} r}{\sqrt{1+G^{2}(r) A_{s}^{2}}} \\
& =\pi D_{0} \int_{0}^{\infty} \frac{(2 / \pi) \exp \left(-2(r / w)^{2}\right) r \mathrm{~d} r}{\sqrt{1+(2 / \pi) \exp \left(-2(r / w)^{2}\right) A_{s}^{2}}} \\
& =\frac{w^{2} D_{0}}{2} \int_{0}^{\infty} \frac{\exp \left(-2(r / w)^{2}\right) \mathrm{d}\left(2(r / w)^{2}\right)}{\sqrt{1+(2 / \pi) \exp \left(-2(r / w)^{2}\right) A_{s}^{2}}} \\
& =\frac{w^{2} D_{0}}{2} \frac{\pi}{A_{s}^{2}}\left[\sqrt{1+\frac{2 A_{s}^{2}}{\pi}}-1\right] .
\end{aligned}
$$


Therefore, the unsaturated population difference $D_{0}$ can be written as

$$
D_{0}=\frac{2 A_{s}^{2}}{\pi w^{2}}\left[\sqrt{1+\frac{2 A_{s}^{2}}{\pi}}-1\right]^{-1} .
$$

The value of the population difference at threshold when lasing just commences is

$$
\begin{aligned}
D_{0, \text { th }} & =\lim _{A_{s} \rightarrow 0} \frac{2 A_{s}^{2}}{\pi w^{2}}\left[\sqrt{1+\frac{2 A_{s}^{2}}{\pi}}-1\right]^{-1} \\
& \cong \frac{2 A_{s}^{2}}{\pi w^{2}}\left[1+\left(\frac{1}{2}\right) \frac{2 A_{s}^{2}}{\pi}-1\right]^{-1}=\frac{2}{w^{2}} .
\end{aligned}
$$

The threshold parameter $\mathfrak{R}$ can be defined as

$$
\mathfrak{R}=\frac{D_{0}}{D_{0, \text { th }}}=\frac{D_{0}}{2} w^{2} .
$$

Equation (10) can be solved for $A_{s}^{2}$ in terms of this threshold parameter as

$$
\begin{gathered}
1=\left(\frac{\Re \pi}{A_{s}^{2}}\right)\left[\sqrt{1+\frac{2 A_{s}^{2}}{\pi}}-1\right], \\
{\left[\frac{A_{s}^{2}}{\Re \pi}+1\right]^{2}=1+\frac{2 A_{s}^{2}}{\pi},} \\
\frac{A_{s}^{4}}{(\Re \pi)^{2}}+\frac{2 A_{s}^{2}}{\Re \pi}+1=1+\frac{2 A_{s}^{2}}{\pi}, \\
\frac{A_{s}^{2}}{(\Re \pi)^{2}}+\frac{2}{\Re \pi}=\frac{2}{\pi}, \\
A_{s}^{2}=2 \Re \pi(\Re-1) .
\end{gathered}
$$

An equivalent result has been given in a previous study of power in steady-state Gaussian-beam lasers [25]. Equation (2) can now be rewritten in terms of the threshold parameter as

$$
\begin{aligned}
\frac{\partial P_{i}(V, r, t)}{\partial t}= & -\gamma\left[P_{i}(V, r, t)+V P_{r}(V, r, t)\right. \\
& \left.+G(r) A(t)\left(\frac{2 \Re}{w^{2}}+D(V, r, t)\right)\right] .
\end{aligned}
$$

\section{STABILITY ANALYSIS}

To analyze the stability behavior of the laser, we assume solutions in the form

$$
\begin{aligned}
& P_{r}(V, r, t)=P_{r s}(V, r)+P_{r}^{\prime}(V, r, t), \\
& P_{i}(V, r, t)=P_{i s}(V, r)+P_{i}^{\prime}(V, r, t), \\
& D(V, r, t)=D_{s}(V, r)+D^{\prime}(V, r, t),
\end{aligned}
$$

$$
A(t)=A_{s}+A^{\prime}(t)
$$

where the primed quantities are assumed to be small and of the same approximate magnitudes relative to each other. With these substitutions, Eqs. (1), (3), (4), and (15) can be rewritten as

$$
\begin{aligned}
\frac{\partial P_{r}^{\prime}(V, r, t)}{\partial t}= & -\gamma\left[\left(P_{r s}(V, r)+P_{r}^{\prime}(V, r, t)\right)-V\left(P_{i s}(V, r)\right.\right. \\
& \left.\left.+P_{i}^{\prime}(V, r, t)\right)\right]=-\gamma\left[P_{r}^{\prime}(V, r, t)-V P_{i}^{\prime}(V, r, t)\right],
\end{aligned}
$$

$$
\begin{aligned}
\frac{\partial P_{i}^{\prime}(V, r, t)}{\partial t}= & -\gamma\left[\left(P_{i s}(V, r)+P_{i}^{\prime}(V, r, t)\right)+V\left(P_{r s}(V, r)\right.\right. \\
& \left.+P_{r}^{\prime}(V, r, t)\right)+G(r)\left(A_{s}+A^{\prime}(t)\right)\left(\frac{2 \Re}{w^{2}}+D_{s}(V, r)\right. \\
& \left.\left.+D^{\prime}(V, r, t)\right)\right] \\
\cong & -\gamma\left[P_{i}^{\prime}(V, r, t)+V P_{r}^{\prime}(V, r, t)+G(r)\right. \\
& \left.\times\left(A_{s} D^{\prime}(V, r, t)+A^{\prime}(t)\left[\frac{2 \Re}{w^{2}}+D_{s}(V, r)\right]\right)\right],
\end{aligned}
$$

$$
\begin{aligned}
\frac{\partial D^{\prime}(V, r, t)}{\partial t}= & -\gamma_{d}\left[\left(D_{s}(V, r)+D^{\prime}(V, r, t)\right)-G(r)\left(A_{s}+A^{\prime}(t)\right)\right. \\
& \left.\times\left(P_{i s}(V, r)+P_{i}^{\prime}(V, r, t)\right)\right] \\
\cong & -\gamma_{d}\left[D^{\prime}(V, r, t)-G(r)\left(A_{s} P_{i}^{\prime}(V, r, t)\right.\right. \\
& \left.\left.+A^{\prime}(t) P_{i s}(V, r)\right)\right]
\end{aligned}
$$

$$
\begin{aligned}
\frac{\mathrm{d} A_{s}^{\prime}(t)}{\mathrm{d} t}= & -\gamma_{c}\left[\left(A_{s}+A^{\prime}(t)\right)+\int_{0}^{\infty} \int_{-\infty}^{\infty} G(r)\left(P_{i s}(V, r)\right.\right. \\
& \left.\left.+P_{i}^{\prime}(V, r, t)\right) \mathrm{d} V r \mathrm{~d} r\right] \\
= & -\gamma_{c}\left[A^{\prime}(t)+\int_{0}^{\infty} \int_{-\infty}^{\infty} G(r) P_{i}^{\prime}(V, r, t) \mathrm{d} V r \mathrm{~d} r\right],
\end{aligned}
$$

where some of the terms involving variables at steady state cancel using Eqs. (5)-(8), and the "approximately equals" signs indicate the dropping of second-order perturbation terms. It is now helpful to introduce the dimensionless decay rate ratios $\delta=\gamma / \gamma_{c}$ and $\rho=\gamma_{d} / \gamma$ together with a normalized time variable $t^{\prime}=\gamma_{c} t$. With these definitions Eqs. (17)-(20) can be rewritten as

$$
\frac{\partial P_{r}^{\prime}\left(V, r, t^{\prime}\right)}{\partial t^{\prime}}=-\delta\left[P_{r}^{\prime}\left(V, r, t^{\prime}\right)-V P_{i}^{\prime}\left(V, r, t^{\prime}\right)\right]
$$




$$
\begin{aligned}
\frac{\partial P_{i}^{\prime}\left(V, r, t^{\prime}\right)}{\partial t^{\prime}}= & -\delta\left[P_{i}^{\prime}\left(V, r, t^{\prime}\right)+V P_{r}^{\prime}\left(V, r, t^{\prime}\right)+G(r)\right. \\
& \left.\times\left(A_{s} D^{\prime}\left(V, r, t^{\prime}\right)+A^{\prime}\left(t^{\prime}\right)\left[\frac{2 \Re}{w^{2}}+D_{s}(V, r)\right]\right)\right], \\
\frac{\partial D^{\prime}\left(V, r, t^{\prime}\right)}{\partial t^{\prime}}= & -\delta \rho\left[D^{\prime}\left(V, r, t^{\prime}\right)-G(r)\left(A_{s} P_{i}^{\prime}\left(V, r, t^{\prime}\right)\right.\right. \\
& \left.\left.+A^{\prime}\left(t^{\prime}\right) P_{i s}(V, r)\right)\right], \\
\frac{\mathrm{d} A^{\prime}\left(t^{\prime}\right)}{\mathrm{d} t^{\prime}}=- & {\left[\begin{array}{c}
\left.A^{\prime}\left(t^{\prime}\right)+\int_{0}^{\infty} \int_{-\infty}^{\infty} G(r) P_{i}^{\prime}\left(V, r, t^{\prime}\right) \mathrm{d} V r \mathrm{~d} r\right] .
\end{array}\right.}
\end{aligned}
$$

Due to the linear nature of Eqs. (21)-(24), we can assume a set of solutions in the form

$$
\begin{gathered}
P_{r}^{\prime}\left(V, r, t^{\prime}\right)=P_{r}^{\prime}(V, r) e^{\lambda t^{\prime}}, \\
P_{i}^{\prime}\left(V, r, t^{\prime}\right)=P_{i}^{\prime}(V, r) e^{\lambda t^{\prime}}, \\
D^{\prime}\left(V, r, t^{\prime}\right)=D^{\prime}(V, r) e^{\lambda t^{\prime}}, \\
A^{\prime}\left(t^{\prime}\right)=A^{\prime} e^{\lambda t^{\prime}},
\end{gathered}
$$

where $\lambda$ is the complex rate constant. With the assumed solutions in Eq. (25) substituted into Eqs. (21)-(24), one obtains

$$
\begin{gathered}
\lambda P_{r}^{\prime}(V, r)=-\delta\left[P_{r}^{\prime}(V, r)-V P_{i}^{\prime}(V, r)\right], \\
(\lambda+\delta) P_{r}^{\prime}(V, r)=\delta V P_{i}^{\prime}(V, r),
\end{gathered}
$$

$$
\begin{gathered}
\lambda P_{i}^{\prime}(V, r)=-\delta\left[P_{i}^{\prime}(V, r)+V P_{r}^{\prime}(V, r)+G(r)\left(A_{s} D^{\prime}(V, r)\right.\right. \\
\left.\left.+A^{\prime}\left[\frac{2 \Re}{w^{2}}+D_{s}(V, r)\right]\right)\right] \\
(\lambda+\delta) P_{i}^{\prime}(V, r)=-\delta\left[V P_{r}^{\prime}(V, r)+G(r)\left(A_{s} D^{\prime}(V, r)\right.\right. \\
\left.\left.+A^{\prime}\left[\frac{2 \Re}{w^{2}}+D_{s}(V, r)\right]\right)\right], \\
(\lambda+\delta \rho) D^{\prime}(V, r)=\delta \rho G(r)\left(A_{s} P_{i}^{\prime}(V, r)+A^{\prime} P_{i s}(V, r)\right), \\
\lambda A^{\prime}=-\left[\begin{array}{l}
\left.A^{\prime}+\int_{0}^{\infty} \int_{-\infty}^{\infty} G(r) P_{i}^{\prime}(V, r) \mathrm{d} V r \mathrm{~d} r\right] \\
(\lambda+1) A^{\prime}=-\int_{0}^{\infty} \int_{-\infty}^{\infty} G(r) P_{i}^{\prime}(V, r) \mathrm{d} V r \mathrm{~d} r .
\end{array}\right.
\end{gathered}
$$

Substituting $P_{r}^{\prime}(V, r)$ from Eq. (26) into Eq. (27) yields

$$
\begin{array}{r}
(\lambda+\delta) P_{i}^{\prime}(V, r)=-\delta\left[V\left(\frac{\delta V}{\lambda+\delta}\right) P_{i}^{\prime}(V, r)+G(r)\left(A_{s} D^{\prime}(V, r)\right.\right. \\
\left.\left.+A^{\prime}\left[\frac{2 \Re}{w^{2}}+D_{s}(V, r)\right]\right)\right] \\
\left(\lambda+\delta+\frac{\delta^{2} V^{2}}{\lambda+\delta}\right) P_{i}^{\prime}(V, r)=-\delta G(r)\left(A_{s} D^{\prime}(V, r)\right. \\
\left.+A^{\prime}\left[\frac{2 \Re}{w^{2}}+D_{s}(V, r)\right]\right)
\end{array}
$$

Combining Eqs. (28) and (30) using Eqs. (7), (9), and (13) leads to

$$
\begin{aligned}
&\left(\lambda+\delta+\frac{\delta^{2} V^{2}}{\lambda+\delta}\right) P_{i}^{\prime}(V, r)=-\delta G(r)\left(A_{s}\left[\frac{\delta \rho G(r)}{(\lambda+\delta \rho)}\left(A_{s} P_{i}^{\prime}(V, r)+A^{\prime} P_{i s}(V, r)\right)\right]+A^{\prime}\left[\frac{2 \Re}{w^{2}}+D_{s}(V, r)\right]\right) \\
&\left(\lambda+\delta+\frac{\delta^{2} V^{2}}{\lambda+\delta}+\frac{\delta^{2} \rho G^{2}(r) A_{s}^{2}}{\lambda+\delta \rho}\right) P_{i}^{\prime}(V, r)=-\frac{\delta^{2} \rho G^{2}(r) A_{s}}{(\lambda+\delta \rho)}\left(-\frac{G(r) A_{s}\left(2 \Re / w^{2}\right)}{1+V^{2}+G^{2}(r) A_{s}^{2}}\right) A^{\prime}-\delta G(r)\left[\frac{2 \Re}{w^{2}}-\frac{G^{2}(r) A_{s}^{2}\left(2 \Re / w^{2}\right)}{1+V^{2}+G^{2}(r) A_{s}^{2}}\right] A^{\prime} \\
&=-\frac{\delta G(r)\left(2 \Re / w^{2}\right) A^{\prime}}{1+V^{2}+G^{2}(r) A_{s}^{2}}\left(1+V^{2}\right)+\left(\frac{\delta G(r)\left(2 \Re / w^{2}\right) A^{\prime}}{1+V^{2}+G^{2}(r) A_{s}^{2}}\right)\left(\frac{\delta \rho G^{2}(r) A_{s}^{2}}{(\lambda+\delta \rho)}\right) \\
&=-\left(\frac{\delta G(r)\left(2 \Re / w^{2}\right) A^{\prime}}{1+V^{2}+G^{2}(r) A_{s}^{2}}\right)\left(1+V^{2}-\frac{\delta \rho G^{2}(r) A_{s}^{2}}{(\lambda+\delta \rho)}\right)
\end{aligned}
$$




$$
P_{i}^{\prime}(V, r)=-\frac{\left(\frac{\delta G(r)\left(2 \Re / w^{2}\right) A^{\prime}}{1+V^{2}+G^{2}(r) A_{s}^{2}}\right)\left(1+V^{2}-\frac{\delta \rho G^{2}(r) A_{s}^{2}}{(\lambda+\delta \rho)}\right)}{\left(\lambda+\delta+\frac{\delta^{2} V^{2}}{(\lambda+\delta)}+\frac{\delta^{2} \rho G^{2}(r) A_{s}^{2}}{(\lambda+\delta \rho)}\right)}
$$

Substituting $P_{i}^{\prime}(V, r)$ from Eq. (31) into Eq. (29) yields

$$
\begin{aligned}
\lambda+1 & =\int_{0}^{\infty} \int_{-\infty}^{\infty}\left[\frac{\left(\frac{\delta G^{2}(r)\left(2 \Re / w^{2}\right)}{1+V^{2}+G^{2}(r) A_{s}^{2}}\right)\left(1+V^{2}-\frac{\delta \rho G^{2}(r) A_{s}^{2}}{\lambda+\delta \rho}\right)}{\left(\lambda+\delta+\frac{\delta^{2} V^{2}}{(\lambda+\delta)}+\frac{\delta^{2} \rho G^{2}(r) A_{s}^{2}}{(\lambda+\delta \rho)}\right)}\right] \mathrm{d} V r \mathrm{~d} r \\
& =\left(\frac{2 \delta \Re}{w^{2}}\right) \int_{0}^{\infty} \int_{-\infty}^{\infty} \frac{(\lambda+\delta) G^{2}(r)}{1+V^{2}+G^{2}(r) A_{s}^{2}} \frac{\left[(\lambda+\delta \rho)\left(1+V^{2}\right)-\delta \rho G^{2}(r) A_{s}^{2}\right]}{\left[(\lambda+\delta \rho)\left[(\lambda+\delta)^{2}+\delta^{2} V^{2}\right]+(\lambda+\delta)\left(\delta^{2} \rho G^{2}(r) A_{s}^{2}\right)\right]} \mathrm{d} V r \mathrm{~d} r \\
& =\left(\frac{2 \delta \Re}{A_{s}^{2}}\right)\left(-\frac{1}{4}\right) \int_{(2 / \pi) A_{s}^{2}}^{0} \frac{(\lambda+\delta)}{1+V^{2}+G^{2}(r) A_{s}^{2}} \frac{\left[(\lambda+\delta \rho)\left[(\lambda+\delta)^{2}+\delta^{2} V^{2}\right]+(\lambda+\delta)\left(\delta^{2} \rho G^{2}(r) A_{s}^{2}\right)\right]}{\left[(\lambda+\delta \rho)\left(1+V^{2}\right)-\delta \rho G^{2}(r) A_{s}^{2}\right] \mathrm{d} V \mathrm{~d}\left(G^{2}(r) A_{s}^{2}\right)} \\
& =\left(\frac{\delta \Re}{2 A_{s}^{2}}\right) \int_{0}^{(2 / \pi) A_{s}^{2}} \int_{-\infty}^{\infty} \frac{(\lambda+\delta)}{1+V^{2}+G^{2}(r) A_{s}^{2}} \frac{\left[(\lambda+\delta \rho)\left[(\lambda+\delta)^{2}+\delta^{2} V^{2}\right]+(\lambda+\delta)\left(\delta^{2} \rho G^{2}(r) A_{s}^{2}\right)\right]}{\left[(\lambda+\delta \rho)\left(1+V^{2}\right)-\delta \rho K\right] \mathrm{d} V \mathrm{~d} K} \\
& =\left(\frac{\delta \Re}{2 A_{s}^{2}}\right) \int_{0}^{(2 / \pi) A_{s}^{2}} \int_{-\infty}^{\infty} \frac{(\lambda+\delta)}{1+V^{2}+K} \frac{[}{\left[(\lambda+\delta \rho)\left[(\lambda+\delta)^{2}+\delta^{2} V^{2}\right]+(\lambda+\delta)\left(\delta^{2} \rho K\right)\right]},
\end{aligned}
$$

where

$$
\begin{gathered}
K=G^{2}(r) A_{s}^{2}, \\
G^{2}(r)=\left(\frac{2}{\pi}\right) \exp \left(\frac{-2 r^{2}}{w^{2}}\right), \\
\frac{\mathrm{d} G^{2}(r)}{\mathrm{d} r}=-\left(\frac{4 r}{w^{2}}\right) G^{2}(r)
\end{gathered}
$$

have been used. Due to the even-function nature of the integrand in Eq. (32) with respect to $V$, one can rewrite Eq. (32) as

$$
\begin{aligned}
\lambda+1 & =\left(\frac{\delta \Re(\lambda+\delta)}{A_{s}^{2}}\right) \int_{0}^{(2 / \pi) A_{s}^{2}} \int_{0}^{\infty} \frac{1}{1+V^{2}+K} \frac{\left[(\lambda+\delta \rho)\left(1+V^{2}\right)-\delta \rho K\right] \mathrm{d} V \mathrm{~d} K}{\left[(\lambda+\delta \rho)\left[(\lambda+\delta)^{2}+\delta^{2} V^{2}\right]+(\lambda+\delta)\left(\delta^{2} \rho K\right)\right]} \\
& =\left(\frac{\delta \Re(\lambda+\delta)}{A_{s}^{2}}\right) \int_{0}^{(2 / \pi) A_{s}^{2}} \int_{0}^{\infty} \frac{1}{1+V^{2}+K} \frac{\left[(\lambda+\delta \rho-\delta \rho K)+(\lambda+\delta \rho) V^{2}\right] \mathrm{d} V \mathrm{~d} K}{\left[(\lambda+\delta \rho)(\lambda+\delta)^{2}+(\lambda+\delta) \delta^{2} \rho K+(\lambda+\delta \rho) \delta^{2} V^{2}\right]} .
\end{aligned}
$$

At this point it is optional whether to perform the $K$ integration or the $V$ integration first. To be specific we start with $V$ and introduce the following variable changes:

$$
\begin{gathered}
A_{1}=\lambda+\delta \rho-\delta \rho K, \\
B_{1}=\lambda+\delta \rho, \\
C_{1}=1+K, \\
D_{1}=(\lambda+\delta \rho)(\lambda+\delta)^{2}+(\lambda+\delta) \delta^{2} \rho K, \\
E_{1}=(\lambda+\delta \rho) \delta^{2} .
\end{gathered}
$$

The $V$ integral in Eq. (36) can then be performed as follows: 


$$
\begin{aligned}
\int_{0}^{\infty} \frac{\left(A_{1}+B_{1} V^{2}\right) \mathrm{d} V}{\left(C_{1}+V^{2}\right)\left(D_{1}+E_{1} V^{2}\right)}= & A_{1} \int_{0}^{\infty} \frac{\mathrm{d} V}{\left(C_{1}+V^{2}\right)\left(D_{1}+E_{1} V^{2}\right)}+B_{1} \int_{0}^{\infty} \frac{V^{2} \mathrm{~d} V}{\left(C_{1}+V^{2}\right)\left(D_{1}+E_{1} V^{2}\right)} \\
= & A_{1}\left[\frac{1}{\left(C_{1} E_{1}-D_{1}\right)}\left\{-\int_{0}^{\infty} \frac{\mathrm{d} V}{C_{1}+V^{2}}+E_{1} \int_{0}^{\infty} \frac{\mathrm{d} V}{\left(D_{1}+E_{1} V^{2}\right)}\right\}\right]+B_{1}\left[\frac { 1 } { ( C _ { 1 } E _ { 1 } - D _ { 1 } ) } \left\{C_{1} \int_{0}^{\infty} \frac{\mathrm{d} V}{C_{1}+V^{2}}\right.\right. \\
& \left.\left.-D_{1} \int_{0}^{\infty} \frac{\mathrm{d} V}{D_{1}+E_{1} V^{2}}\right\}\right], \\
= & A_{1}\left[\frac{1}{\left(C_{1} E_{1}-D_{1}\right)}\left\{-\frac{\pi}{2 \sqrt{C_{1}}}+\frac{\pi}{2 \sqrt{D_{1} / E_{1}}}\right\}\right]+B_{1}\left[\frac{1}{\left(C_{1} E_{1}-D_{1}\right)}\left\{C_{1} \frac{\pi}{2 \sqrt{C_{1}}}-\left(\frac{D_{1}}{E_{1}}\right) \frac{\pi}{2 \sqrt{D_{1} / E_{1}}}\right\}\right] \\
= & \frac{\pi}{2\left(C_{1} E_{1}-D_{1}\right)}\left[A_{1}\left(\sqrt{\frac{E_{1}}{D_{1}}}-\frac{1}{\sqrt{C_{1}}}\right)+B_{1}\left(\sqrt{C_{1}}-\sqrt{\frac{D_{1}}{E_{1}}}\right)\right] .
\end{aligned}
$$

Equations (14), (36), and (38) may be combined to obtain

$$
\begin{aligned}
\lambda+1 & =\left(\frac{\delta \Re(\lambda+\delta)}{A_{s}^{2}}\right) \int_{0}^{(2 / \pi) A_{s}^{2}} \frac{\pi}{2\left(C_{1} E_{1}-D_{1}\right)}\left[A_{1}\left(\sqrt{\frac{E_{1}}{D_{1}}}-\frac{1}{\sqrt{C_{1}}}\right)+B_{1}\left(\sqrt{C_{1}}-\sqrt{\frac{D_{1}}{E_{1}}}\right)\right] \mathrm{d} K \\
& =\left(\frac{\delta \Re(\lambda+\delta)}{2 \Re \pi(\Re-1)}\right)\left(\frac{\pi}{2}\right) \int_{0}^{4 \Re(\Re-1)} \frac{1}{\left(C_{1} E_{1}-D_{1}\right)}\left[A_{1}\left(\sqrt{\frac{E_{1}}{D_{1}}}-\frac{1}{\sqrt{C_{1}}}\right)+B_{1}\left(\sqrt{C_{1}}-\sqrt{\frac{D_{1}}{E_{1}}}\right)\right] \mathrm{d} K \\
& =\left(\frac{\delta(\lambda+\delta)}{4(\Re-1)}\right) \int_{0}^{4 \Re(\Re-1)} \frac{1}{\left(C_{1} E_{1}-D_{1}\right)}\left[A_{1}\left(\sqrt{\frac{E_{1}}{D_{1}}}-\frac{1}{\sqrt{C_{1}}}\right)+B_{1}\left(\sqrt{C_{1}}-\sqrt{\frac{D_{1}}{E_{1}}}\right)\right] \mathrm{d} K .
\end{aligned}
$$

Using again the definitions in Eq. (37), Eq. (39) becomes the result

$$
\begin{aligned}
0= & \lambda+1-\left(\frac{\delta(\lambda+\delta)}{4(\Re-1)}\right) \int_{0}^{4 \Re(\Re-1)}\left[\frac{1}{(1+K)(\lambda+\delta \rho) \delta^{2}-\left((\lambda+\delta \rho)(\lambda+\delta)^{2}+(\lambda+\delta) \delta^{2} \rho K\right)}\right. \\
& \times\left\{(\lambda+\delta \rho-\delta \rho K)\left[\left(\frac{(\lambda+\delta \rho) \delta^{2}}{(\lambda+\delta \rho)(\lambda+\delta)^{2}+(\lambda+\delta) \delta^{2} \rho K}\right)^{1 / 2}-\frac{1}{\sqrt{1+K}}\right]\right. \\
& \left.\left.+(\lambda+\delta \rho)\left[\sqrt{1+K}-\left(\frac{(\lambda+\delta \rho)(\lambda+\delta)^{2}+(\lambda+\delta) \delta^{2} \rho K}{(\lambda+\delta \rho) \delta^{2}}\right)^{1 / 2}\right]\right\}\right] d K .
\end{aligned}
$$

The next step in this analysis could be to carry out the remaining integration over the parameter $K$. This process is possible in closed form and leads to an implicit analytic equation for the rate constant $\lambda$. However, the integration is tedious, and with a computer it is simpler to analyze Eq. (40) directly. In general, the complex rate constant $\lambda$ in Eq. (40) consists of a real and an imaginary part. A negative value of the real part of $\lambda$ means that a small perturbation of the steady-state solution will decay with time. This indicates that the laser system is in a stable mode of operation. On the other hand, a positive value of the real part of $\lambda$ means that a small perturbation of the steady-state solution will increase with time indicating that the system is unstable. Exactly at the instability threshold, the real part of $\lambda$ is zero. Equation (40) has been programmed and solved for the instability threshold using a two-dimensional secant method in the complex plane.
The results of the calculations of Eq. (40) for a Gaussian-beam electromagnetic field are the stability curves shown in Fig. 1. Each curve in the figure represents the stability boundary below which the laser pro-

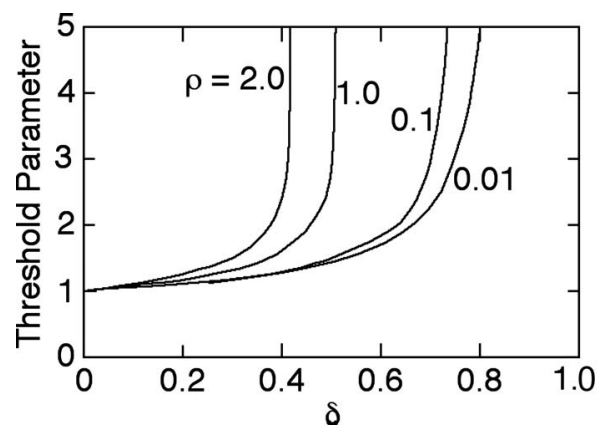

Fig. 1. Type 1 stability boundaries for inhomogeneously broadened unidirectional ring-laser oscillators with Gaussian-beam electromagnetic fields. 


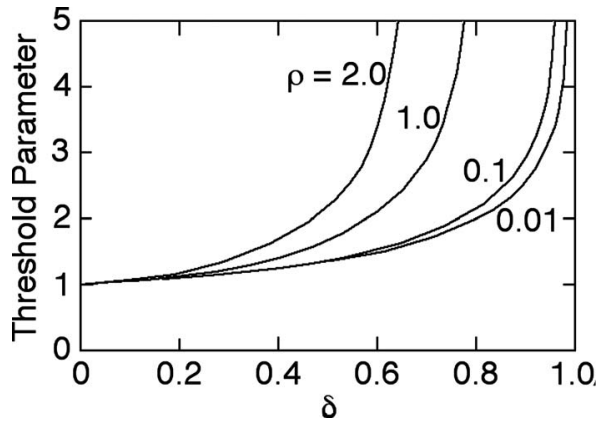

Fig. 2. Type 1 stability boundaries for inhomogeneously broadened unidirectional ring-laser oscillators with uniform-planewave electric fields (after [21]).

duces a cw output field. Above each curve small perturbations of the cw solution increase with time indicating that the laser is unstable with respect to spontaneous pulsations. The instability boundaries for the corresponding plane-wave electromagnetic field are shown in Fig. 2 [21]. A comparison of these results shows that, for the same values of $\delta$ and $\rho$, a laser with a constant spot-size Gaussian field requires higher values of the threshold parameter to reach the instability threshold compared to a laser with a plane-wave field. This indicates that the Gaussian field envelope, in effect, helps stabilize the laser system. It should be noted, however, that for values of the parameter $\delta$ below about 0.3 the instability thresholds for both the plane-wave and Gaussian-beam field models are close to unity. As reviewed in [21], the value of $\delta$ for a typical $3.51 \mu \mathrm{m}$ xenon laser is about 0.033 , and from the data reviewed in [26] the value of $\delta$ for a $3.39 \mu \mathrm{m}$ helium-neon laser is about 0.22 . Thus, even with the inclusion of a Gaussian field distribution, the stability thresholds for these well-known spontaneously pulsing systems are close to the ordinary lasing thresholds.

\section{CONCLUSION}

We have investigated the stability of a semiclassical laser model for inhomogeneously broadened unidirectional ring-laser oscillators with constant-spot-size $\mathrm{TEM}_{00}$ Gaussian fields. Type 1 stability boundaries were obtained by applying a linear stability analysis to this model. A comparison of the stability boundaries from the present study with those for lasers with uniform-planewave fields shows that, for the same values of laser parameters, the Gaussian field distribution helps in stabilizing the lasers against small perturbations by raising the type 1 instability threshold and by narrowing the range of parameter values for which instabilities can be observed. These effects are, however, much less significant than for other laser types. Low-threshold spontaneous pulsations are readily observed experimentally in inhomogeneously broadened lasers that simultaneously include the stabilizing effects of Gaussian-mode transverse fields, standing-wave longitudinal fields, and some degree of intracavity field focusing.

\section{REFERENCES}

1. N. B. Abraham, L. A. Lugiato, and L. M. Narducci, "Overview of instabilities in laser systems," J. Opt. Soc. Am. B 2, 7-14 (1985) and references.

2. H. Haken, "Analogy between higher instabilities in fluids and lasers," Phys. Lett. A 53, 77-78 (1975).

3. L. A. Lugiato and M. Milani, "Disappearance of laser instabilities in a Gaussian cavity mode," Opt. Commun. 46, 57-60 (1983).

4. S. Stuut and M. Sargent III, "Effects of Gaussian-beam averaging on phase conjugation and beat-frequency spectroscopy," J. Opt. Soc. Am. B 1, 95-101 (1984).

5. L. A. Lugiato and M. Milani, "Effects of Gaussian-beam averaging on laser instabilities," J. Opt. Soc. Am. B 2, 15-17 (1985).

6. L. A. Lugiato, R. J. Horowicz, and G. Strini, "Instabilities in passive and active optical systems with a Gaussian transverse intensity profile," Phys. Rev. A 30, 1366-1376 (1984).

7. D. Y. Tang, C. O. Weiss, E. Roldán, and C. J. de Valcárcel, "Deviation from Lorenz-type dynamics of an $\mathrm{NH}_{3}$ ring laser," Opt. Commun. 89, 47-53 (1992).

8. C. O. Weiss, R. Vilaseca, N. B. Abraham, R. Corbalin, E. Roldán, G. J. de Valcárcel, J. Pujol, U. Hübner, and D. Y. Tang, "Models, predictions, and experimental measurements of far-infrared $\mathrm{NH}_{3}$-laser dynamics and comparisons with the Lorenz-Haken model," Appl. Phys. B 61, 223-242 (1995).

9. C. P. Smith and R. Dykstra, "Lorenz-like chaos in a Gaussian mode laser with a radially dependent gain," Opt. Commun. 117, 107-110 (1995).

10. C. P. Smith and R. Dykstra, "Observation in the two-level spatial Maxwell-Bloch model of the anomalously large first peak as seen in experimental Lorenz-like spiral chaos from the ${ }^{15} \mathrm{NH}_{3}$ laser," Opt. Commun. 129, 69-74 (1996).

11. J. F. Urchueguia, G. J. de Valcárcel, and E. Roldán, "Laser instabilities in a Gaussian cavity mode with Gaussian pump profile," J. Opt. Soc. Am. B 15, 1512-1520 (1998).

12. J. F. Urchueguia, G. J. de Valcárcel, E. Roldán, and F. Prati, "Transverse effects in ring fiber laser multimode instabilities," Phys. Rev. A 62, 041801 (2000).

13. E. Roldán, G. J. de Valcárcel, J. F. Urchueguia, and J. M. Guerra, "Observability of the Risken-NummedalGraham-Haken instability in Nd:YAG lasers," J. Opt. Soc. Am. B 20, 816-824 (2003).

14. L. W. Casperson, "Spontaneous coherent pulsations in laser oscillators," IEEE J. Quantum Electron. QE-14, 756-761 (1978).

15. L. W. Casperson, Laser Physics, J. D. Harvey and D. F. Walls, eds., Vol. 182 of Springer Lecture Notes in Physics (Springer-Verlag, 1983), pp. 88-106.

16. J. Bentley and N. B. Abraham, "Mode-pulling, modesplitting and pulsing in high-gain lasers," J. Opt. Soc. Am. 70, 1622 (1980).

17. J. Bentley and N. B. Abraham, "Mode-pulling, modesplitting, and pulsing in a high gain He-Xe laser," Opt. Commun. 41, 52-56 (1982).

18. M. Maeda and N. B. Abraham, "Measurements of modesplitting self-pulsing in a single-mode, Fabry-Perot laser," Phys. Rev. A 26, 3395-3403 (1982).

19. R. S. Gioggia and N. B. Abraham, "Single-mode self-pulsing instabilities at the Lamb dip of a He-Ne $3.39 \mu \mathrm{m}$ laser," Opt. Commun. 47, 278-282 (1983).

20. R. S. Gioggia and N. B. Abraham, "Anomalous mode pulling, instabilities, and chaos in a single mode, standingwave 3.39- $\mu \mathrm{m}$ He-Ne laser," Phys. Rev. A 29, 1304-1309 (1984).

21. P. Chenkosol and L. W. Casperson, "Stability criteria for spontaneously pulsing gas lasers," J. Opt. Soc. Am. B 26, 939-945 (2009).

22. L. W. Casperson, "Spontaneous coherent pulsations in ringlaser oscillators," J. Opt. Soc. Am. B 2, 62-72 (1985).

23. P. Chenkosol and L. W. Casperson, "Spontaneous coherent pulsations in standing-wave laser oscillators: stability 
criteria for homogeneous broadening," J. Opt. Soc. Am. B 10, 817-826 (1993).

24. L. W. Casperson, "Spontaneous coherent pulsations in ringlaser oscillators: stability criteria," J. Opt. Soc. Am. 2, 993-997 (1985) and references.
25. L. W. Casperson, "Laser power calculations: sources of error," Appl. Opt. 19, 422-434 (1980).

26. P. Chenkosol and L. W. Casperson, "Spontaneous coherent pulsations in $3.39 \mu \mathrm{m}$ He-Ne standing-wave laser oscillators,” J. Opt. Soc. Am. B 20, 2539-2547 (2003). 